\title{
Vertex operator solutions to the discrete KP-hierarchy*
}

\author{
M. Adler ${ }^{\dagger}$ P. van Moerbeke
}

August 24, 1998

\section{Contents}

$\begin{array}{llll}1 & \text { The KP } \tau \text {-functions, Grassmannians and a residue formula } & 7\end{array}$

2 The existence of a $\tau$-vector and the discrete KP bilinear identity

13

3 Sequences of $\tau$-functions, flags and the discrete KP equation 18

4 Discrete KP-solutions generated by vertex operators 23

5 Example of vertex generated solutions: the $q$-KP equation 24

Vertex operators, which are disguised Darboux maps, transform solutions of the KP equation into new ones. In this paper, we show that the bi-infinite

*The final version appeared in: Comm. Math. Phys., 203, 185-210 (1999)

†Department of Mathematics, Brandeis University, Waltham, Mass 02454, USA. Email: adler@math.brandeis.edu. The support of a National Science Foundation grant \# DMS-9503246 is gratefully acknowledged.

†Department of Mathematics, Université de Louvain, 1348 Louvain-la-Neuve, Belgium and Brandeis University, Waltham, Mass 02454, USA. E-mail: vanmoerbeke@geom.ucl.ac.be and @math.brandeis.edu. The support of a National Science Foundation grant \# DMS-9503246, a Nato, a FNRS and a Francqui Foundation grant is gratefully acknowledged. 
sequence obtained by Darboux transforming an arbitrary KP solution recursively forward and backwards, yields a solution to the discrete KP-hierarchy. The latter is a KP hierarchy where the continuous space $x$-variable gets replaced by a discrete $n$-variable. The fact that these sequences satisfy the discrete KP hierarchy is tantamount to certain bilinear relations connecting the consecutive KP solutions in the sequence. At the Grassmannian level, these relations are equivalent to a very simple fact, which is the nesting of the associated infinite-dimensional planes (flag). The discrete KP hierarchy can thus be viewed as a container for an entire ensemble of vertex or Darboux generated KP solutions.

It turns out that many new and old systems lead to such discrete (semiinfinite) solutions, like sequences of soliton solutions, with more and more solitons, sequences of Calogero-Moser systems, having more and more particles, just to mention a few examples; this is developped in [3]. In this paper, as an other example, we show that the $q$-KP hierarchy maps, via a kind of Fourier transform, into the discrete KP hierarchy, enabling us to write down a very large class of solutions to the $q$-KP hierarchy. This was also reported in a brief note with E. Horozov $\llbracket$.

Given the shift operator $\Lambda=\left(\delta_{i, j-1}\right)_{i, j \in \mathbf{Z}}$, consider the Lie algebra

$$
\mathcal{D}=\left\{\sum_{-\infty<i \ll \infty} a_{i} \Lambda^{i}, a_{i} \text { diagonal operators }\right\}=\mathcal{D}_{-}+\mathcal{D}_{+}
$$

with the usual splitting $\mathcal{D}=\mathcal{D}_{-}+\mathcal{D}_{+}$, into subalgebras

$$
\mathcal{D}_{+}=\left\{\sum_{0 \leq i \ll \infty} a_{i} \Lambda^{i} \in \mathcal{D}\right\}, \mathcal{D}_{-}=\left\{\sum_{-\infty<i<0} a_{i} \Lambda^{i} \in \mathcal{D}\right\} .
$$

The discrete KP-hierarchy equations

$$
\frac{\partial L}{\partial t_{n}}=\left[\left(L^{n}\right)_{+}, L\right], \quad n=1,2, \ldots
$$

are deformations of an infinite matrix

$$
L=\sum_{-\infty<i \leq 0} a_{i}(t) \Lambda^{i}+\Lambda \in \mathcal{D}, \quad \text { with } t=\left(t_{1}, t_{2}, \ldots\right) \in \mathbf{C}^{\infty}
$$


If we represent $L$ as a dressing up of $\Lambda$ by a wave operator $S \in I+\mathcal{D}_{-}$

$$
L=S \Lambda S^{-1}=W \Lambda W^{-1}, \quad W=S e^{\sum_{1}^{\infty} t_{i} \Lambda^{i}},
$$

then the $L$-deformations are induced by $S$-deformations and $W$-deformations:

$$
\frac{\partial S}{\partial t_{n}}=-\left(L^{n}\right)_{-} S, \quad \frac{\partial W}{\partial t_{n}}=\left(L^{n}\right)_{+} W, \quad n=1,2, \ldots ;
$$

In terms of vectors

$$
\chi(z)=\left(z^{n}\right)_{n \in \mathbf{Z}}, \quad \chi^{*}(z)=\chi\left(z^{-1}\right),
$$

such that $z \chi(z)=\Lambda \chi(z), \quad z \chi^{*}(z)=\Lambda^{\top} \chi^{*}(z)$, let us define wave and adjoint wave vectors $\Psi(t, z)$ and $\Psi^{*}(t, z)$

$$
\Psi(t, z)=W \chi(z) \text { and } \Psi^{*}(t, z)=\left(W^{-1}\right)^{\top} \chi^{*}(z) .
$$

We find, using (0.5), (0.8), (0.6), that

$$
\begin{aligned}
L \Psi(t, z) & =z \Psi(t, z) & L^{\top} \Psi^{*}(t, z)=z \Psi^{*}(t, z), \\
\frac{\partial \Psi}{\partial t_{n}} & =\left(L^{n}\right)_{+} \Psi & \frac{\partial \Psi^{*}}{\partial t_{n}}=-\left(\left(L^{n}\right)_{+}\right)^{\top} \Psi^{*} .
\end{aligned}
$$

Theorem 0.1 If $L$ satisfies the Toda lattice, then the wave vectors $\Psi(t, z)$ and $\Psi^{*}(t, z)$ can be expressed in terms of one sequence of $\tau$-functions $\tau(n, t):=$ $\tau_{n}\left(t_{1}, t_{2}, \ldots\right), \quad n \in \mathbf{Z}$, to wit:

$$
\begin{gathered}
\Psi(t, z)=\left(e^{\sum_{1}^{\infty} t_{i} z^{i}} \psi(t, z)\right)_{n \in \mathbf{Z}}=\left(\frac{\tau_{n}\left(t-\left[z^{-1}\right]\right)}{\tau_{n}(t)} e^{\sum_{1}^{\infty} t_{i} z^{i}} z^{n}\right)_{n \in \mathbf{Z}}, \\
\Psi^{*}(t, z)=\left(e^{-\sum_{1}^{\infty} t_{i} z^{i}} \psi^{*}(t, z)\right)_{n \in \mathbf{Z}}=\left(\frac{\tau_{n+1}\left(t+\left[z^{-1}\right]\right)}{\tau_{n+1}(t)} e^{-\sum_{1}^{\infty} t_{i} z^{i}} z^{-n}\right)_{n \in \mathbf{Z}},
\end{gathered}
$$

satisfying the bilinear identity

$$
\oint_{z=\infty} \Psi_{n}(t, z) \Psi_{m}^{*}\left(t^{\prime}, z\right) \frac{d z}{2 \pi i z}=0
$$


for all $n>m$. It follows that

$$
\begin{gathered}
\Psi=W \chi(z)=e^{\sum_{1}^{\infty} t_{i} z^{i}} S \chi(z) \\
\Psi^{*}=\left(W^{\top}\right)^{-1} \chi^{*}(z)=e^{-\sum_{1}^{\infty} t_{i} z^{i}}\left(S^{-1}\right)^{\top} \chi^{*}(z),
\end{gathered}
$$

with

$$
S=\sum_{0}^{\infty} \frac{p_{n}(-\tilde{\partial}) \tau(t)}{\tau(t)} \Lambda^{-n} \quad \text { and } \quad S^{-1}=\sum_{0}^{\infty} \Lambda^{-n} \tilde{\Lambda}\left(\frac{p_{n}(\tilde{\partial}) \tau(t)}{\tau(t)}\right) .
$$

Then $L^{k}$ has the following expression in terms of $\tau$-function $\}^{2}$,

$$
L^{k}=\sum_{\ell=0}^{\infty} \operatorname{diag}\left(\frac{p_{\ell}(\tilde{\partial}) \tau_{n+k-\ell+1} \circ \tau_{n}}{\tau_{n+k-\ell+1} \tau_{n}}\right)_{n \in \mathbf{Z}} \Lambda^{k-\ell}
$$

with the $\tau_{n}$ 's satisfying

$$
\left(\frac{\partial}{\partial t_{k}}-\sum_{r=0}^{\ell-1}(\ell-r) p_{r}(-\tilde{\partial}) p_{k-r}(\tilde{\partial})\right) \tau_{n} \circ \tau_{n-\ell}=0, \quad \text { for } \ell, k=1,2,3, \ldots
$$

and

$$
\left(\frac{1}{2} \frac{\partial^{2}}{\partial t_{1} \partial t_{k}}-p_{k+1}(\tilde{\partial})\right) \tau_{n} \circ \tau_{n}=0, \quad \text { for } k=1,2,3, \ldots
$$

Remark: Equation (0.14) reads

$$
\begin{aligned}
L^{k}= & \Lambda^{k}+\left(\frac{\partial}{\partial t_{1}} \log \frac{\tau_{n+k}}{\tau_{n}}\right)_{n \in \mathbf{Z}} \Lambda^{k-1}+\ldots \\
& +\left(\frac{\partial}{\partial t_{k}} \log \frac{\tau_{n+1}}{\tau_{n}}\right)_{n \in \mathbf{Z}} \Lambda^{0}+\left(\frac{\partial^{2}}{\partial t_{1} \partial t_{k}} \log \tau_{n}\right)_{n \in \mathbf{Z}} \Lambda^{-1}+\ldots
\end{aligned}
$$

\footnotetext{
${ }^{1}$ In an expression, like $S=\sum a^{(n)} \Lambda^{n}, a^{(n)}=\operatorname{diag}\left(a_{k}^{(n)}\right)_{k \in \mathbf{Z}}$ and $(\tilde{\Lambda} a)_{k}=a_{k+1} \Lambda^{0}$.

${ }^{2}$ where the $p_{\ell}$ are elementary Schur polynomials and where $p_{\ell}(\tilde{\partial}) f \circ g$ refers to the usual Hirota operation, to be defined in section 1.
} 
With each component of the wave vector $\Psi$, or, what is the same, with each component of the $\tau$-vector, we associate a sequence of infinite-dimensional planes in the Grassmannian $G r^{(n)}$

$$
\begin{aligned}
\mathcal{W}_{n} & =\operatorname{span}_{\mathbf{C}}\left\{\left(\frac{\partial}{\partial t_{1}}\right)^{k} \Psi_{n}(t, z), \quad k=0,1,2, \ldots\right\} \\
& =e^{\sum_{1}^{\infty} t_{i} z^{i}} \operatorname{span}_{\mathbf{C}}\left\{\left(\frac{\partial}{\partial t_{1}}+z\right)^{k} \psi_{n}(t, z), \quad k=0,1,2, \ldots\right\} \\
& =: e^{\sum_{1}^{\infty} t_{i} z^{i}} \mathcal{W}_{n}^{t} .
\end{aligned}
$$

Note that the plane $z^{-n} \mathcal{W}_{n} \in G r^{(0)}$ has so-called virtual genus zero, in the terminology of [12]; in particular, this plane contains an element of order $1+O\left(z^{-1}\right)$. Setting $\{f, g\}=f^{\prime} g-f g^{\prime}$ for ${ }^{\prime}=\partial / \partial t_{1}$, we have the following statement:

Theorem 0.2 The following six statements are equivalent

(i) The discrete KP-equations (0.3)

(ii) $\Psi$ and $\Psi^{*}$, with the proper asymptotic behaviour, given by (0.8), satisfy the bilinear identities for all $t, t^{\prime} \in \mathbf{C}^{\infty}$

$$
\oint_{z=\infty} \Psi_{n}(t, z) \Psi_{m}^{*}\left(t^{\prime}, z\right) \frac{d z}{2 \pi i z}=0, \quad \text { for all } n>m
$$

(iii) the $\tau$-vector satisfies the following bilinear identities for all $n>m$ and $t, t^{\prime} \in \mathbf{C}^{\infty}$ :

$$
\oint_{z=\infty} \tau_{n}\left(t-\left[z^{-1}\right]\right) \tau_{m+1}\left(t^{\prime}+\left[z^{-1}\right]\right) e^{\sum_{1}^{\infty}\left(t_{i}-t_{i}^{\prime}\right) z^{i}} z^{n-m-1} d z=0
$$

(iv) The components $\tau_{n}$ of a $\tau$-vector correspond to a flag of planes in $G r$,

$$
\ldots \supset \mathcal{W}_{n-1} \supset \mathcal{W}_{n} \supset \mathcal{W}_{n+1} \supset \ldots
$$

(v) A sequence of KP- $\tau$-functions $\tau_{n}$ satisfying the equations

$$
\left\{\tau_{n}\left(t-\left[z^{-1}\right]\right), \tau_{n+1}(t)\right\}+z\left(\tau_{n}\left(t-\left[z^{-1}\right]\right) \tau_{n+1}(t)-\tau_{n+1}\left(t-\left[z^{-1}\right]\right) \tau_{n}(t)\right)=0
$$


(vi) A sequence of KP- $\tau$-functions $\tau_{n}$ satisfying equations (0.14) for $\ell=1$, i.e.,

$$
\left(\frac{\partial}{\partial t_{k}}-p_{k}(\tilde{\partial})\right) \tau_{n+1} \circ \tau_{n}=0 \text { for } k=2,3, \ldots \text { and } n \in \mathbf{Z} \text {. }
$$

Remark: The 2-Toda lattice, studied in [14], amounts to two coupled 1-Toda lattices or discrete KP-hierarchies, thus introducing two sets of times $t_{n}$ 's and $s_{n}$ 's. Actually, every 1-Toda lattice can naturally be extended to a 2-Toda lattice; this is the content of Theorem 3.4.

How to construct discrete KP-solutions. A wide class of examples of discrete KP-solutions is given in section 4 by the following construction, involving the simple vertex operators,

$$
X(t, z):=e^{\sum_{1}^{\infty} t_{i} z^{i}} e^{-\sum_{1}^{\infty} \frac{z^{-i}}{i} \frac{\partial}{\partial t_{i}}}
$$

which are disguised Darboux transformations acting on KP $\tau$-functions. We now state:

Theorem 0.3 Consider an arbitrary $\tau$-function for the KP equation and a family of weights ..., $\nu_{-1}(z) d z, \nu_{0}(z) d z, \nu_{1}(z) d z, \ldots$ on $\mathbf{R}$. The infinite sequence of $\tau$-functions: $\tau_{0}=\tau$ and, for $n>0$,

$$
\begin{gathered}
\tau_{n}:=\left(\int X(t, \lambda) \nu_{n-1}(\lambda) d \lambda \ldots \int X(t, \lambda) \nu_{0}(\lambda) d \lambda\right) \tau(t), \\
\tau_{-n}:=\left(\int X(-t, \lambda) \nu_{-n}(\lambda) d \lambda \ldots \int X(-t, \lambda) \nu_{-1}(\lambda) d \lambda\right) \tau(t),
\end{gathered}
$$

form a discrete KP- $\tau$-vector, i.e., the bi-infinite matrix

$$
L=\sum_{\ell=0}^{\infty} \operatorname{diag}\left(\frac{p_{\ell}(\tilde{\partial}) \tau_{n+2-\ell} \circ \tau_{n}}{\tau_{n+2-\ell} \tau_{n}}\right)_{n \in \mathbf{Z}} \Lambda^{1-\ell}
$$

satisfies the discrete KP hierarchy (0.3).

As an interesting special case of this situation, we study in section 6 the $q-K P$ equation. 
A wide variety of examples are captured by this construction, like $q$-approximations to KP, discussed in section 5, but also soliton formulas, matrix integrals, certain integrals leading to band matrices, the Calogero-Moser system and others, discussed in [3].

Remark: A semi-infinite discrete KP-hierarchy with $\tau_{0}(t)=1$ is equivalent to a bi-infinite discrete KP-hierarchy with $\tau_{-n}(t)=\tau_{n}(-t)$ and $\tau_{0}(t)=1$; this also amounts to $\mathcal{W}_{-n}=\mathcal{W}_{n}^{*}$, with $\mathcal{W}_{0}=\mathcal{H}_{+}$. In such cases, one only keeps the lower right hand corner of $L$, while the lower left hand corner completely vanishes.

\section{The KP $\tau$-functions, Grassmannians and a residue formula}

As is well known [5], the bilinear identity

$$
\oint_{z=\infty} \Psi(t, z) \Psi^{*}(t, z) d z=0
$$

together with the asymptotics

$$
\Psi(t, z)=e^{\sum_{1}^{\infty} t_{i} z^{i}}\left(1+O\left(\frac{1}{z}\right)\right), \Psi^{*}(t, z)=e^{-\sum_{1}^{\infty} t_{i} z^{i}}\left(1+O\left(\frac{1}{z}\right)\right)
$$

force $\Psi, \Psi^{*}$ to be expressible in terms of $\tau$-functions

$$
\Psi(t, z)=e^{\sum_{1}^{\infty} t_{i} z^{i}} \frac{\tau\left(t-\left[z^{-1}\right]\right)}{\tau(t)}, \Psi^{*}(t, z)=e^{-\sum_{1}^{\infty} t_{i} z^{i}} \frac{\tau\left(t+\left[z^{-1}\right]\right)}{\tau(t)}
$$

moreover the KP $\tau$-functions satisfy the differential Fay identity', for all $y, z \in \mathbf{C}$, as shown in [1, 15]:

$$
\begin{aligned}
& \left\{\tau\left(t-\left[y^{-1}\right]\right), \tau\left(t-\left[z^{-1}\right]\right)\right\} \\
& \quad+(y-z)\left(\tau\left(t-\left[y^{-1}\right]\right) \tau\left(t-\left[z^{-1}\right]\right)-\tau(t) \tau\left(t-\left[y^{-1}\right]-\left[z^{-1}\right]\right)=0 .\right.
\end{aligned}
$$

In fact this identity characterizes the $\tau$-function, as shown in $[13$.

$$
{ }^{3}\{f, g\}:=\frac{\partial f}{\partial t_{1}} g-f \frac{\partial g}{\partial t_{1}} .
$$


From (1.1), it follows that

$$
\begin{gathered}
0=\oint \tau\left(t-a-\left[z^{-1}\right]\right) \tau\left(t+a+\left[z^{-1}\right]\right) e^{-2 \sum_{1}^{\infty} a_{i} z^{i}} \frac{d z}{2 \pi i} \\
=\sum_{k=1}^{\infty} a_{k}\left(\frac{\partial^{2}}{\partial t_{1} \partial t_{k}}-2 p_{k+1}(\tilde{\partial})\right) \tau \circ \tau+O\left(a^{2}\right)
\end{gathered}
$$

The Hirota notation used here is the following: Given a polynomial $p\left(\frac{\partial}{\partial t_{1}}, \frac{\partial}{\partial t_{2}}, \ldots\right)$ in $\frac{\partial}{\partial t_{i}}$, define the symbol

$$
p\left(\frac{\partial}{\partial t_{1}}, \frac{\partial}{\partial t_{2}}, \ldots\right) f \circ g(t):=\left.p\left(\frac{\partial}{\partial u_{1}}, \frac{\partial}{\partial u_{2}}, \ldots\right) f(t+u) g(t-u)\right|_{u=0},
$$

and

$$
\tilde{\partial}_{t}:=\left(\frac{\partial}{\partial t_{1}}, \frac{1}{2} \frac{\partial}{\partial t_{2}}, \frac{1}{3} \frac{\partial}{\partial t_{3}}, \ldots\right) .
$$

For future use, we state the following proposition shown in [四]:

Proposition 1.1 Consider $\tau$-functions $\tau_{1}$ and $\tau_{2}$, the corresponding wave functions

$$
\Psi_{j}=e^{\sum_{i \geq 1} t_{i} z^{i}} \frac{\tau_{j}\left(t-\left[z^{-1}\right]\right)}{\tau_{j}(t)}=e^{\sum_{i \geq 1} t_{i} z^{i}}\left(1+O\left(z^{-1}\right)\right)
$$

and the associated infinite-dimensional planes, as points in the Grassmannian Gr,

$$
\tilde{\mathcal{W}}_{i}=\operatorname{span}\left\{\left(\frac{\partial}{\partial t_{1}}\right)^{k} \Psi_{i}(t, z), \text { for } k=0,1,2, \ldots\right\} \text { with } \tilde{\mathcal{W}}_{i}^{t}=\tilde{\mathcal{W}}_{i} e^{-\sum_{1}^{\infty} t_{k} z^{k}}
$$

then the following statements are equivalent

(i) $z \tilde{\mathcal{W}}_{2} \subset \tilde{\mathcal{W}}_{1}$

(ii) $z \Psi_{2}(t, z)=\frac{\partial}{\partial t_{1}} \Psi_{1}(t, z)-\alpha \Psi_{1}(t, z)$, for some function $\alpha=\alpha(t)$;

(iii)

$$
\left\{\tau_{1}\left(t-\left[z^{-1}\right]\right), \tau_{2}(t)\right\}+z\left(\tau_{1}\left(t-\left[z^{-1}\right]\right) \tau_{2}(t)-\tau_{2}\left(t-\left[z^{-1}\right]\right) \tau_{1}(t)\right)=0 .
$$


When (i), (ii) or (iii) holds, $\alpha(t)$ is given by

$$
\alpha(t)=\frac{\partial}{\partial t_{1}} \log \frac{\tau_{2}}{\tau_{1}} .
$$

Proof: To prove that $(\mathrm{i}) \Rightarrow(\mathrm{ii})$, the inclusion $z \tilde{\mathcal{W}}_{2} \subset \tilde{\mathcal{W}}_{1}$, hence $z \tilde{\mathcal{W}}_{2}^{t} \subset$ $\tilde{\mathcal{W}}_{1}^{t}$, implies by $(0.16)$ that

$$
z \psi_{2}(t, z)=z\left(1+O\left(z^{-1}\right)\right) \in \tilde{\mathcal{W}}_{1}^{t}
$$

must be a linear combination

$$
z \psi_{2}=\frac{\partial \psi_{1}}{\partial x}+z \psi_{1}-\alpha(t) \psi_{1} \text {, and thus } z \Psi_{2}=\frac{\partial}{\partial t_{1}} \Psi_{1}-\alpha(t) \Psi_{1} .
$$

The expression (1.8) for $\alpha(t)$ follows from equating the $z^{0}$-coefficient in (ii), upon using the $\tau$-function representation (1.6). To show that (ii) $\Rightarrow$ (i), note that

$$
z \Psi_{2}=\frac{\partial}{\partial t_{1}} \Psi_{1}-\alpha \Psi_{1} \in \tilde{\mathcal{W}}_{1}
$$

and taking $t_{1}$-derivatives, we have

$$
z\left(\frac{\partial}{\partial t_{1}}\right)^{j} \Psi_{2}=\left(\frac{\partial}{\partial t_{1}}\right)^{j+1} \Psi_{1}+\beta_{1}\left(\frac{\partial}{\partial t_{1}}\right)^{j} \Psi_{1}+\cdots+\beta_{j+1} \Psi_{1}
$$

for some $\beta_{1}, \cdots, \beta_{j+1}$ depending on $t$ only; this implies the inclusion (i). The equivalence (ii) $\Longleftrightarrow$ (iii) follows from a straightforward computation using the $\tau$-function representation (1.6) of (ii) and the expression for $\alpha(t)$.

Lemma 1.2 The following integral along a clockwise circle in the complex plane encompassing $z=\infty$ and $z=\alpha^{-1}$, can be evaluated as follows

$$
\begin{aligned}
& \oint_{z=\infty} f\left(t+[\alpha]-\left[z^{-1}\right]\right) g\left(t-[\alpha]+\left[z^{-1}\right]\right) \frac{z^{m+1}}{\left(z-\alpha^{-1}\right)^{2}} \frac{d z}{2 \pi i z} \\
= & \alpha^{1-m} \sum_{k=1}^{\infty} \alpha^{k}\left(-\frac{\partial}{\partial t_{k}}+\sum_{r=0}^{m-1}(m-r) p_{r}(-\tilde{\partial}) p_{k-r}(+\tilde{\partial})\right) f \circ g .
\end{aligned}
$$

\footnotetext{
${ }^{4} \psi_{i}$ is the same as $\Psi_{i}$, but without the exponential.
} 
Proof: By the residue theorem, the integral above is the sum of residue at $z=\infty$ and at $z=\alpha^{-1}$ :

$$
\begin{aligned}
& \oint_{z=\infty} f\left(t+[\alpha]-\left[z^{-1}\right]\right) g\left(t-[\alpha]+\left[z^{-1}\right]\right) \frac{z^{m+1}}{\left(z-\alpha^{-1}\right)^{2}} \frac{d z}{2 \pi i z} \\
= & \left.\frac{1}{(m-1) !}\left(\frac{d}{d u}\right)^{m-1} f(t+[\alpha]-[u]) g(t-[\alpha]+[u]) \frac{1}{\left(1-u \alpha^{-1}\right)^{2}}\right|_{u=0} \\
& -\left.\frac{d}{d z} z^{m} f\left(t+[\alpha]-\left[z^{-1}\right]\right) g\left(t-[\alpha]+\left[z^{-1}\right]\right)\right|_{z=\alpha^{-1}}
\end{aligned}
$$

Evaluating each of the pieces requires a few steps.

\section{Step 1.}

$$
\left.\frac{1}{k !}\left(\frac{d}{d u}\right)^{k} f(t+[\alpha]-[u]) g(t-[\alpha]+[u])\right|_{u=0}=\sum_{\ell=0}^{\infty} \alpha^{\ell} p_{k}(-\tilde{\partial}) p_{\ell}(\tilde{\partial}) f \circ g .
$$

At first note

$$
\left.\left(\frac{d}{d u}\right)^{k} F([u])\right|_{u=0}=k ! p_{k}\left(\tilde{\partial}_{s}\right) F(s)
$$

and, by (1.5) and (1.12),

$$
\begin{aligned}
\left.\frac{1}{k !}\left(\frac{d}{d u}\right)^{k} f(t+[u]) g(t-[u])\right|_{u=0} & =p_{k}(\tilde{\partial}) f \circ g \\
& =p_{k}(-\tilde{\partial}) g \circ f \\
& =\sum_{i+j=k} p_{i}(-\tilde{\partial}) g \cdot p_{j}(\tilde{\partial}) f
\end{aligned}
$$

Indeed

$$
\begin{aligned}
& \left.\frac{1}{k !}\left(\frac{d}{d u}\right)^{k} f(t+[\alpha]-[u]) g(t-[\alpha]+[u])\right|_{u=0} \\
= & \left.p_{k}\left(\tilde{\partial}_{s}\right) g(t-[\alpha]+s) f(t+[\alpha]-s)\right|_{s=0}, \quad u \operatorname{using}(1.12) \\
= & \left.p_{k}\left(\tilde{\partial}_{s}\right) \sum_{\ell=0}^{\infty} \alpha^{\ell} p_{\ell}\left(\tilde{\partial}_{t}\right) f(t-s) \circ g(t+s)\right|_{s=0}, \quad u \operatorname{using}(1.13)
\end{aligned}
$$




$$
\begin{aligned}
& =\left.\sum_{\ell=0}^{\infty} \alpha^{\ell} p_{k}\left(\tilde{\partial}_{s}\right) p_{\ell}\left(\tilde{\partial}_{w}\right) f(t+w-s) g(t-w+s)\right|_{s=w=0}, \quad \text { expressing Hirota, } \\
& =\left.\sum_{\ell=0}^{\infty} \alpha^{\ell} p_{k}\left(\tilde{\partial}_{s}\right) p_{\ell}\left(-\tilde{\partial}_{w}\right) f(t-w-s) g(t+w+s)\right|_{s=w=0}, \quad \text { flipping signs, } \\
& =\left.\sum_{\ell=0}^{\infty} \alpha^{\ell} p_{k}\left(\tilde{\partial}_{v}\right) p_{\ell}\left(-\tilde{\partial}_{v}\right) f(t-v) g(t+v)\right|_{v=0} \\
& =\sum_{\ell=0}^{\infty} \alpha^{\ell} p_{k}(-\tilde{\partial}) p_{\ell}(\tilde{\partial}) f \circ g, \quad \operatorname{using}(1.13) .
\end{aligned}
$$

Step 2. Residue at $\infty$.

Note

$$
\left.\left(\frac{d}{d u}\right)^{\ell}\left(\frac{1}{1-u \alpha^{-1}}\right)^{2}\right|_{u=0}=\left.\left(\frac{d}{d u}\right)^{\ell} \sum_{i=1}^{\infty} i\left(u \alpha^{-1}\right)^{i-1}\right|_{u=0}=(\ell+1) ! \alpha^{-\ell}
$$

then we find

$$
\begin{aligned}
& \left.\frac{1}{(m-1) !}\left(\frac{d}{d u}\right)^{m-1} f(t+[\alpha]-[u]) g(t-[\alpha]+[u]) \frac{1}{\left(1-u \alpha^{-1}\right)^{2}}\right|_{u=0} \\
= & \frac{1}{(m-1) !} \sum_{r=0}^{m-1}\left(\begin{array}{c}
m-1 \\
r
\end{array}\right)\left(\frac{d}{d u}\right)^{r} f(t+[\alpha]-[u]) g(t-[\alpha]+[u]) \\
= & \sum_{r=0}^{m-1}(m-r) \sum_{\ell=0}^{\infty} \alpha^{\ell-m+r+1} p_{r}(-\tilde{\partial}) p_{\ell}(\tilde{\partial}) f \circ g, \quad \text { using step } 1 \text { and }(1.14) \\
= & m \alpha^{1-m} f(t) g(t)+\alpha^{1-m} \sum_{k=1}^{\infty} \alpha^{k} \sum_{r=0}^{m}(m-r) p_{r}(-\tilde{\partial}) p_{k-r}(\tilde{\partial}) f \circ g, \quad \text { using } p_{0}=1 .
\end{aligned}
$$

Step 3. Residue at $z=\alpha^{-1}$.

$$
\begin{aligned}
& \left.\frac{d}{d z} z^{m} f\left(t+[\alpha]-\left[z^{-1}\right]\right) g\left(t-[\alpha]+\left[z^{-1}\right]\right)\right|_{z=\alpha^{-1}} \\
& =-\left.u^{2} \frac{d}{d u} u^{-m} f(t+[\alpha]-[u]) g(t-[\alpha]+[u])\right|_{u=\alpha}
\end{aligned}
$$


Adler-van Moerbeke:Discrete KP August 24, $1998 \quad \S 1$, p.12

$$
\begin{aligned}
& =m \alpha^{-m+1} f(t) g(t)-\left.\alpha^{2-m} \frac{d}{d u} f(t+[\alpha]-[u]) g(t-[\alpha]+[u])\right|_{u=\alpha} \\
& =m \alpha^{1-m} f(t) g(t)+\sum_{k=1}^{\infty} \alpha^{1-m+k} \frac{\partial}{\partial t_{k}} f \circ g, \quad \text { by explicit differentiation. }
\end{aligned}
$$

Finally, putting step 2 and step 3 in (1.11) yields Lemma 1.2.

Lemma 1.3 The Hirota symbol acts as follows on functions $f\left(t_{1}, t_{2}, \ldots\right)$ and $g\left(t_{1}, t_{2}, \ldots\right)$ :

$$
\frac{1}{f g} \frac{\partial^{n}}{\partial t_{1} \ldots \partial t_{n}} f \circ g=\text { a polynomial } P_{n} \text { in }\left\{\begin{array}{l}
\frac{\partial^{k}}{\partial t_{i_{1}} \ldots \partial t_{i_{k}}} \log \frac{f}{g} \quad \text { for } k \text { odd } \\
\frac{\partial^{k}}{\partial t_{i_{1} \ldots \partial t_{i_{k}}}} \log f g \quad \text { for } k \text { even }
\end{array}\right.
$$

over all subsets $\left\{i_{1}, \ldots, i_{k}\right\} \subset\{1, \ldots, n\}$. Upon granting degree 1 to each partial in $t_{i}$, the polynomial $P_{n}$ is homogeneous of degree $n$.

Proof: By induction, we assume the statement to be valid for an $\mathrm{Hi}$ rota symbol, involving $\ell$ partials, and we prove the statement for a symbol involving $\ell+1$ partials:

$$
\begin{aligned}
& \frac{1}{f g} \frac{\partial}{\partial t_{\ell+1}} \frac{\partial^{\ell}}{\partial t_{1} \ldots \partial t_{\ell}} f(t) \circ g(t) \\
= & \left.\frac{1}{f g} \frac{\partial}{\partial u_{\ell+1}} f(t+u) g(t-u) \frac{\frac{\partial^{\ell}}{\partial t_{1} \ldots \partial t_{\ell}} f(t+u) \circ g(t-u)}{f(t+u) g(t-u)}\right|_{u=0} \\
= & \left(\frac{\partial}{\partial t_{\ell+1}} \log \frac{f}{g}\right) \frac{1}{f g} \frac{\partial^{\ell}}{\partial t_{1} \ldots \partial t_{\ell}} f(t+u) \circ g(t-u) \\
& +\left.\frac{\partial}{\partial u_{\ell+1}} P\left(\ldots, \frac{\partial^{m}}{\partial t_{i_{1}} \ldots t_{i_{m}}} \log \frac{f(t+u)}{g(t-u)}, \ldots, \frac{\partial^{n}}{\partial t_{j_{1}} \ldots \partial t_{j_{n}}} \log f(t+u) g(t-u), \ldots\right)\right|_{u=0},
\end{aligned}
$$

where $m$ is odd and $n$ even. The result follows from the simple computation:

$$
\left.\frac{\partial}{\partial u_{\ell+1}} \frac{\partial^{m}}{\partial t_{i_{1}} \ldots \partial t_{i_{m}}} \log \frac{f(t+u)}{g(t-u)}\right|_{u=0}=\frac{\partial^{m+1}}{\partial t_{i_{1}} \ldots \partial t_{i_{m}} . \partial t_{\ell+1}} \log f(t) g(t)
$$


Adler-van Moerbeke:Discrete KP August 24, $1998 \quad \S 2$, p.13

$$
\left.\frac{\partial}{\partial u_{\ell+1}} \frac{\partial^{n}}{\partial t_{i_{1}} \ldots \partial t_{i_{n}}} \log f(t+u) g(t-u)\right|_{u=0}=\frac{\partial^{n+1}}{\partial t_{i_{1}} \ldots \partial t_{i_{n}} . \partial t_{\ell+1}} \log \frac{f(t)}{g(t)}
$$

Remark: The induction formula (1.18) can be made into an explicit formula for $P_{n}$, involving partitions of the set $\{1,2, \ldots, n\}$.

\section{The existence of a $\tau$-vector and the discrete KP bilinear identity}

Before proving Theorem 0.1, we shall need two lemmas, which are analogues of basic lemmas in the theory of differential operators. So the main purpose of this section is threefold, namely, to prove the bilinear identities for the wave and adjoint wave vectors, to prove the existence of a $\tau$-vector and finally to give a closed form for $L^{k}$.

Lemma 2.1 For z-independent $U, V \in \mathcal{D}$, the following matrix identities hold

$$
U V=\oint_{z=\infty} U \chi(z) \otimes V^{\top} \chi^{*}(z) \frac{d z}{2 \pi i z}
$$

Proof: Set

$$
U=\sum_{\alpha} u_{\alpha} \Lambda^{\alpha} \quad \text { and } \quad V=\sum_{\beta} \Lambda^{\beta} v_{\beta}
$$

where $u_{\alpha}$ and $v_{\alpha}$ are diagonal matrices. To prove (2.1), it suffices to compare the $(i, j)$-entries on each side. On the left side of $(2.1)$, we have

$$
\begin{aligned}
(U V)_{i j} & =\left(\sum_{\alpha, \beta} u_{\alpha} \Lambda^{\alpha+\beta} v_{\beta}\right)_{i j} \\
& =\sum_{\alpha, \beta} u_{\alpha}(i)\left(\Lambda^{\alpha+\beta}\right)_{i j} v_{\beta}(j) \\
& =\sum_{\substack{\alpha, \beta \\
\alpha+\beta=j-i}} u_{\alpha}(i) v_{\beta}(j) .
\end{aligned}
$$

\footnotetext{
${ }^{5}(A \otimes B)_{i j}=A_{i} B_{j}$ and remember $\chi^{*}(z)=\chi\left(z^{-1}\right)$. The contour in the integration below runs clockwise about $\infty$; i.e., opposite to the usual orientation.
} 
Adler-van Moerbeke:Discrete KP August 24, $1998 \quad \S 2$, p.14

On the right side of (2.1), we have

$$
\begin{aligned}
\oint_{z=\infty} & (U \chi(z))_{i}\left(V^{\top} \chi\left(z^{-1}\right)\right)_{j} \frac{d z}{2 \pi i z} \\
= & \oint_{z=\infty}\left(\sum_{\alpha} u_{\alpha} z^{\alpha} \chi(z)\right)_{i}\left(\sum_{\beta} v_{\beta} z^{\beta} \chi\left(z^{-1}\right)\right)_{j} \frac{d z}{2 \pi i z} \\
= & \oint_{z=\infty} \sum_{\alpha, \beta} u_{\alpha}(i) v_{\beta}(j) z^{\alpha+\beta+i-j} \frac{d z}{2 \pi i z} \\
= & \sum_{\substack{\alpha, \beta \\
\alpha+\beta=j-i}} u_{\alpha}(i) v_{\beta}(j),
\end{aligned}
$$

establishing (2.1).

Lemma 2.2 For $W(t)$ a wave operator of the discrete KP-hierarchy,

$$
W(t) W^{-1}\left(t^{\prime}\right) \in \mathcal{D}_{+}, \quad \forall t, t^{\prime} .
$$

Proof: Setting $h\left(t, t^{\prime}\right)=W(t) W^{-1}\left(t^{\prime}\right)$, compute from $(0.6)$

$$
\frac{\partial h}{\partial t_{n}}=\left(L^{n}(t)\right)_{+} h, \quad \frac{\partial h}{\partial t_{n}^{\prime}}=-h\left(L^{n}\left(t^{\prime}\right)\right)_{+},
$$

since $h(t, t)=I \in \mathcal{D}_{+}$, it follows that $h\left(t, t^{\prime}\right)$ evolves in $\mathcal{D}_{+}$.

Consider the wave function, already defined in the introduction, and the adjoint wave function:

$$
\begin{gathered}
\Psi(t, z)=W \chi(z)=e^{\sum_{1}^{\infty} t_{i} z^{i}} S \chi(z)=e^{\sum t_{i} z^{i}}\left(z^{n}+\sum_{i<n} s_{i}(n) z^{i}\right)_{n \in \mathbf{Z}} \\
\Psi^{*}(t, z)=\left(W^{-1}\right)^{\top} \chi^{*}(z)=e^{-\sum_{1}^{\infty} t_{i} z^{i}\left(S^{-1}\right)^{\top} \chi^{*}(z)} \\
=e^{-\sum t_{i} z^{i}}\left(z^{-n}+\sum_{i<-n} s_{i}^{*}(n) z^{i}\right)_{n \in \mathbf{Z}}
\end{gathered}
$$

Proof of Theorem 0.1:

Step 1: Setting

$$
U:=W(t) \quad \text { and } \quad V^{\top}:=\left(W^{-1}\left(t^{\prime}\right)\right)^{\top}
$$


Adler-van Moerbeke:Discrete KP August 24, $1998 \quad \S 2$, p.15

in formula (2.1) of Lemma 2.1, and using formula (0.8) of $\Psi$ and $\Psi^{*}$ in terms of $W$, one finds for all $t, t^{\prime} \in \mathbf{C}^{\infty}$,

$$
W(t) W\left(t^{\prime}\right)^{-1}=\oint_{z=\infty} \Psi(t, z) \otimes \Psi^{*}\left(t^{\prime}, z\right) \frac{d z}{2 \pi i z}
$$

But, according to Lemma $2.2, W(t) W\left(t^{\prime}\right)^{-1} \in \mathcal{D}_{+}$and thus (2.5) is uppertriangular, yielding

$$
\oint_{z=\infty} \Psi_{n}(t, z) \Psi_{m}^{*}\left(t^{\prime}, z\right) \frac{d z}{2 \pi i z}=0 \quad \text { for all } n>m .
$$

Defining

$$
\begin{aligned}
& \Phi_{n}(t, z):=z^{-n} \Psi_{n}(t, z)=e^{\sum t_{i} z^{i}}\left(1+O\left(z^{-1}\right)\right) \\
& \Phi_{n}^{*}(t, z):=z^{n-1} \Psi_{n-1}^{*}(t, z)=e^{-\sum t_{i} z^{i}}\left(1+O\left(z^{-1}\right)\right),
\end{aligned}
$$

upon using the asymptotics (0.8), we have, by setting $m=n-1$ in (2.6)

$$
\oint_{z=\infty} \Phi_{n}(t, z) \Phi_{n}^{*}\left(t^{\prime}, z\right) d z=\oint_{z=\infty} \Psi_{n}(t, z) \Psi_{n-1}^{*}\left(t^{\prime}, z\right) \frac{d z}{z}=0
$$

From the KP-theory, there exists a $\tau$-function $\tau_{n}(t)$ for each $n$, such that

$$
\Phi_{n}(t, z)=e^{\sum t_{i} z^{i}} \frac{\tau_{n}\left(t-\left[z^{-1}\right]\right)}{\tau_{n}(t)}, \quad \Phi_{n}^{*}(t, z)=e^{-\sum t_{i} z^{i}} \frac{\tau_{n}\left(t+\left[z^{-1}\right]\right)}{\tau_{n}(t)}
$$

yielding the $\tau$-function representation $(0.10)$ for $\Psi_{n}$ and $\Psi_{n}^{*}$.

Step 2: The following holds for $n \in \mathbf{Z}$ :

$$
\begin{gathered}
\left(\frac{1}{2} \frac{\partial^{2}}{\partial t_{1} \partial t_{k}}-p_{k+1}(\tilde{\partial})\right) \tau_{n} \circ \tau_{n}=0, \quad \text { for } k=1,2,3, \ldots \\
\left(\frac{\partial}{\partial t_{k}}-\sum_{r=0}^{\ell-1}(\ell-r) p_{r}(-\tilde{\partial}) p_{k-r}(\tilde{\partial})\right) \tau_{n} \circ \tau_{n-\ell}=0, \quad \text { for } \ell, k=1,2,3, \ldots
\end{gathered}
$$

Indeed the bilinear identity (2.6), upon setting $m=n-\ell-1$, shifting $t \mapsto t+[\alpha], t^{\prime} \mapsto t-[\alpha]$, using the $\tau$-function representation (0.10) of $\Psi$ and 
$\Psi^{*}$, and lemma 1.2 with $m=\ell$, yield

$$
\begin{aligned}
0 & =-\alpha^{2} \oint_{z=\infty} \Psi_{n}(t+[\alpha], z) \Psi_{n-\ell-1}^{*}(t-[\alpha], z) \frac{d z}{2 \pi i z} \tau_{n}(t+[\alpha]) \tau_{n-\ell}(t-[\alpha]) \\
& =-\oint_{z=\infty} \tau_{n}\left(t+[\alpha]-\left[z^{-1}\right]\right) \tau_{n-\ell}\left(t-[\alpha]+\left[z^{-1}\right]\right) e^{2 \sum_{1}^{\infty}(\alpha z)^{i} / i} \alpha^{2} z^{\ell+1} \frac{d z}{2 \pi i z} \\
& =\alpha^{1-\ell} \sum_{k=1}^{\infty} \alpha^{k}\left(\frac{\partial}{\partial t_{k}}-\sum_{r=0}^{\ell-1}(\ell-r) p_{r}(-\tilde{\partial}) p_{k-r}(\tilde{\partial})\right) \tau_{n} \circ \tau_{n-\ell},
\end{aligned}
$$

establishing the second relation of (2.8). As for the first one, set $m=n-1$, $t \mapsto t-a$ and $t^{\prime} \mapsto t+a$ in the bilinear identity, and use (1.4), thus yielding (0.14).

Step 3: To check the formulas (0.12) for $S$, compute

$$
\begin{aligned}
e^{\sum_{1}^{\infty} t_{i} z^{i} S \chi(z)} & =: \Psi(t, z) \\
& =e^{\sum_{1}^{\infty} t_{i} z^{i}} \frac{\tau\left(t-\left[z^{-1}\right]\right)}{\tau(t)} \chi(z) \quad(\text { by }(0.10)) \\
& =e^{\sum_{1}^{\infty} t_{i} z^{i}} \sum_{n=0}^{\infty} \frac{p_{n}(-\tilde{\partial}) \tau(t)}{\tau(t)} z^{-n} \chi(z) \\
& =e^{\sum_{1}^{\infty} t_{i} z^{i}} \sum_{0}^{\infty} \frac{p_{n}(-\tilde{\partial}) \tau(t)}{\tau(t)} \Lambda^{-n} \chi(z) .
\end{aligned}
$$

Similarly one checks the formula for $S^{-1}$ using the formulas for $\Psi^{*}(t, z)$ in terms of $S^{-1}$ and $\tau(t)$. Finally to check the formula (0.13) for $L^{k}$, use the formulas (0.12) for $S$ and $S^{-1}$ (for $\tilde{\Lambda}$, see footnote 1$)$ :

$$
\begin{aligned}
L^{k} & =S \Lambda^{k} S^{-1} \\
& =\sum_{i, j \geq 0}^{\infty} \frac{p_{i}(-\tilde{\partial}) \tau}{\tau} \Lambda^{-i-j+k}\left(\tilde{\Lambda} \frac{p_{j}(\tilde{\partial}) \tau}{\tau}\right) \\
& =\sum_{i, j \geq 0}^{\infty} \frac{p_{i}(-\tilde{\partial}) \tau}{\tau}\left(\tilde{\Lambda}^{-i-j+k+1} \frac{p_{j}(\tilde{\partial}) \tau}{\tau}\right) \Lambda^{-i-j+k} \\
& =\sum_{\ell \geq 0}\left(\sum_{\substack{i, j \geq 0 \\
i+j=\ell}} \frac{\left.p_{i}(-\tilde{\partial}) \tau_{n} p_{j}(\tilde{\partial}) \tau_{n+k-\ell+1}\right)}{\tau_{n} \tau_{n+k-\ell+1}}\right)_{n \in \mathbf{Z}} \Lambda^{k-\ell}
\end{aligned}
$$

$$
{ }^{6} e^{m \sum_{1}^{\infty}(\alpha z)^{i} / i}=(1-\alpha z)^{-m}
$$


Adler-van Moerbeke:Discrete KP August 24, 1998 §3, p.17

$$
=\sum_{\ell \geq 0}\left(\frac{p_{\ell}(\tilde{\partial}) \tau_{n+k-\ell+1} \circ \tau_{n}}{\tau_{n+k-\ell+1} \tau_{n}}\right)_{n \in \mathbf{Z}} \Lambda^{k-\ell} \quad(\operatorname{using}(1.13))
$$

yielding (0.13) and (0.15), upon noting,

$$
\begin{aligned}
\operatorname{coef}_{\Lambda^{k-1}} L^{k} & =\left(\frac{p_{1}(\tilde{\partial}) \tau_{n+k} \circ \tau_{n}}{\tau_{n+k} \tau_{n}}\right)_{n \in \mathbf{Z}}=\left(\frac{\partial}{\partial t_{1}} \log \frac{\tau_{n+k}}{\tau_{n}}\right)_{n \in \mathbf{Z}} \\
\operatorname{coef}_{\Lambda^{0}} L^{k} & =\left(\frac{p_{k}(\tilde{\partial}) \tau_{n+1} \circ \tau_{n}}{\tau_{n+1} \tau_{n}}\right)_{n \in \mathbf{Z}}=\left(\frac{\partial}{\partial t_{k}} \log \frac{\tau_{n+1}}{\tau_{n}}\right)_{n \in \mathbf{Z}} \text { by }(2.8) \\
\operatorname{coef}_{\Lambda^{-1}} L^{k} & =\left(\frac{p_{k+1}(\tilde{\partial}) \tau_{n} \circ \tau_{n}}{\tau_{n} \tau_{n}}\right)_{n \in \mathbf{Z}}=\left(\frac{\partial^{2}}{\partial t_{1} \partial t_{k}} \log \tau_{n}\right)_{n \in \mathbf{Z}}, \quad \text { by }(2.7),
\end{aligned}
$$

concluding the proof of the Theorem 0.1 .

Corollary 2.3 Setting $\gamma(t):=(\tilde{\Lambda} \tau(t) / \tau(t))$, the wave operator $W(t)$ for the discrete KP-hierarchy has the following property

$$
\left(W(t) W^{-1}\left(t^{\prime}\right)\right)_{-}=0, \quad\left(W(t) W^{-1}\left(t^{\prime}\right)\right)_{0}=\frac{\gamma(t)}{\gamma\left(t^{\prime}\right)} .
$$

Proof: That $h\left(t, t^{\prime}\right)=W(t) W^{-1}\left(t^{\prime}\right) \in \mathcal{D}_{+}$was shown in Lemma 2.2. Concerning its diagonal $h_{0}$, we deduce from (2.3) that

$$
\frac{\partial}{\partial t_{k}} \log h_{0}=\left(L^{k}(t)\right)_{0}, \quad \frac{\partial}{\partial t_{k}} \log h_{0}=-\left(L^{k}\left(t^{\prime}\right)\right)_{0}, \quad \text { with } h_{0}(t, t)=I .
$$

Note that $\gamma(t) / \gamma\left(t^{\prime}\right)$ satisfies the same differential equations as $h_{0}(t)$ with the same initial condition, upon using (0.15):

$$
\begin{aligned}
& \left(\frac{\partial}{\partial t_{k}} \log \frac{\gamma(t)}{\gamma\left(t^{\prime}\right)}\right)_{n}=\frac{\partial}{\partial t_{k}} \log \frac{\tau_{n+1}(t)}{\tau_{n}(t)}=L^{k}(t)_{n n} \\
& \left(\frac{\partial}{\partial t_{k}^{\prime}} \log \frac{\gamma(t)}{\gamma\left(t^{\prime}\right)}\right)_{n}=-\frac{\partial}{\partial t_{k}^{\prime}} \log \frac{\tau_{n+1}\left(t^{\prime}\right)}{\tau_{n}\left(t^{\prime}\right)}=-L^{k}\left(t^{\prime}\right)_{n n}
\end{aligned}
$$

with $\gamma(t) /\left.\gamma\left(t^{\prime}\right)\right|_{t=t^{\prime}}=I$.

\footnotetext{
${ }^{7} M_{0}:=$ diagonal part of $M$.
} 
Adler-van Moerbeke:Discrete KP August 24, 1998 33, p.18

\section{Sequences of $\tau$-functions, flags and the dis- crete KP equation}

In this section, we prove Theorem 0.2 ; it will be broken up into three propositions: the first one is very similar to the analogous statement for the KP theory (see [5, 15). One could make an argument unifying both cases, in the context of Lie theory. The second statement uses Grassmannian technology.

Proposition 3.1 The following equivalences $(i) \Longleftrightarrow$ (ii) $\Longleftrightarrow$ (iii) hold.

Proof: (i) $\Rightarrow$ (ii) was already shown in Theorem 0.1. Regarding the converse (ii) $\Rightarrow$ (i), we show vectors $\Psi(t, z)$ and $\Psi^{*}(t, z)$ having the asymptotics (0.8) and satisfying the bilinear identity (ii) are discrete KP-hierarchy vectors.

The point of the proof is to show that the matrices $S$ and $T^{t} \in I+\mathcal{D}_{-}$ defined through

$$
\Psi(t, z)=: e^{\sum_{1}^{\infty} t_{i} z^{i}} S \chi(z), \quad \Psi^{*}(t, z)=: e^{-\sum_{1}^{\infty} t_{i} z^{i}} T \chi^{*}(z)
$$

satisfy the vector fields (0.6) with $T^{t}=S^{-1}$.

Step 1. $T^{t}=S^{-1}$.

Assuming the bilinear identities (assumption (ii) of Theorem 0.2),

$$
\begin{aligned}
0 & =\left(\oint_{z=\infty} \Psi(t, z) \otimes \Psi^{*}(t, z) \frac{d z}{2 \pi i z}\right)_{-} \\
& =\left(\oint_{z=\infty} e^{\sum_{1}^{\infty} t_{i} z^{i}} S \chi(z) \otimes e^{-\sum_{1}^{\infty} t_{i} z^{i}} T \chi\left(z^{-1}\right) \frac{d z}{2 \pi i z}\right)_{-} \\
& =\left(S T^{\top}\right)_{-}, \quad \text { by }(2.1)
\end{aligned}
$$

but since $S, T^{t} \in I+\mathcal{D}_{-}, S T^{t}=I$, yielding $T^{t}=S^{-1}$.

Step 2. $W(t) W^{-1}\left(t^{\prime}\right) \in \mathcal{D}_{+}$, upon defining $W(t):=S(t) e^{\sum t_{i} \Lambda^{i}}$.

According to the bilinear identity, the left hand side of

$$
\oint_{z=\infty} \Psi(t, z) \otimes \Psi^{*}\left(t^{\prime}, z\right) \frac{d z}{2 \pi i z}
$$


Adler-van Moerbeke:Discrete KP August 24, $1998 \quad \S 3$, p.19

$$
\begin{aligned}
& =\oint_{z=\infty} e^{\sum t_{i} z^{i}} S \chi(z) \otimes e^{-\sum_{1}^{\infty} t_{i}^{\prime} z^{i}}\left(S^{-1}\right)^{\top} \chi\left(z^{-1}\right) \frac{d z}{2 \pi i z} \\
& =\oint_{z=\infty} S(t) e^{\sum t_{i} \Lambda^{i}} \chi(z) \otimes\left(S^{-1}\left(t^{\prime}\right)\right)^{\top} e^{-\sum t_{i}^{\prime} \Lambda^{\top-i}} \chi\left(z^{-1}\right) \frac{d z}{2 \pi i z} \\
& =S(t) e^{\sum t_{i} \Lambda^{i}} e^{-\sum t_{i}^{\prime} \Lambda^{i}} S^{-1}\left(t^{\prime}\right), \quad \text { using Lemma } 2.1 \\
& =W(t) W^{-1}\left(t^{\prime}\right) ;
\end{aligned}
$$

belongs to $\mathcal{D}_{+}$, and hence so is the right hand side.

\section{Step 3.}

$$
\begin{aligned}
\left(\frac{\partial}{\partial t_{n}}-\left(L^{n}\right)_{+}\right) \Psi(t, z) & =\left(\frac{\partial}{\partial t_{n}}-\left(L^{n}\right)_{+}\right) S \chi(z) e^{\sum_{1}^{\infty} t_{i} z^{i}} \\
& =\left(\frac{\partial S}{\partial t_{n}}-\left(L^{n}\right)_{+} S+S z^{n}\right) \chi(z) e^{\sum_{1}^{\infty} t_{i} z^{i}} \\
& =\left(\frac{\partial S}{\partial t_{n}}-\left(L^{n}\right)_{+} S+S \Lambda^{n}\left(S^{-1} S\right)\right) \chi(z) e^{\sum_{1}^{\infty} t_{i} z^{i}} \\
& =\left(\frac{\partial S}{\partial t_{n}}-\left(L^{n}\right)_{+} S+L^{n} S\right) \chi(z) e^{\sum_{1}^{\infty} t_{i} z^{i}} \\
& \left.=\left(\frac{\partial S}{\partial t_{n}}+\left(L^{n}\right)_{-} S\right)\right) \chi(z) e^{\sum_{1}^{\infty} t_{i} z^{i}}
\end{aligned}
$$

Step 4. From $W(t) W^{-1}\left(t^{\prime}\right) \in \mathcal{D}_{+}$, since $\mathcal{D}_{+}$is an algebra, deduce

$$
\begin{aligned}
\mathcal{D}_{+} & \left.\ni\left(\left(\frac{\partial}{\partial t_{n}}-\left(L^{n}\right)_{+}\right) W(t)\right) W^{-1}\left(t^{\prime}\right)\right|_{t^{\prime}=t} \\
& =\oint_{z=\infty}\left(\frac{\partial}{\partial t_{n}}-\left(L^{n}\right)_{+}\right) \Psi(t, z) \otimes \Psi^{*}(t, z) \frac{d z}{2 \pi i z}, \quad \text { by step } 2 \\
& =\oint_{z=\infty}\left(\frac{\partial S(t)}{\partial t_{n}}+\left(L^{n}\right)_{-} S(t)\right) \chi(z) e^{\sum_{1}^{\infty} t_{i} z^{i}} \otimes\left(S^{\top}(t)\right)^{-1} \chi\left(z^{-1}\right) e^{-\sum_{1}^{\infty} t_{i} z^{i}} \frac{d z}{2 \pi i z}, \\
& =\left(\frac{\partial S(t)}{\partial t_{n}}+\left(L^{n}\right)_{-} S(t)\right) S(t)^{-1}, \quad \text { by step } 3
\end{aligned}
$$

and thus, since $S \in I+\mathcal{D}_{-}$and $\mathcal{D}_{-}$is an algebra,

$$
\left(\frac{\partial S(t)}{\partial t_{n}}+\left(L^{n}\right)_{-} S(t)\right) S(t)^{-1} \in \mathcal{D}_{+} \cap \mathcal{D}_{-}=0
$$


Adler-van Moerbeke:Discrete KP August 24, 1998 §3, p.20

therefore, we have the discrete KP-hierarchy equations on $S$

$$
\frac{\partial S(t)}{\partial t_{n}}+\left(L^{n}\right)_{-} S=0, \quad n=1,2, \ldots
$$

and on $L=S \Lambda S^{-1}$

$$
\frac{\partial L}{\partial t_{n}}=\left[-\left(L^{n}\right)_{-}, L\right]
$$

ending the proof that (ii) $\Rightarrow$ (i).

Finally (ii) $\Longleftrightarrow$ (iii) upon using the equivalence (i) $\Longleftrightarrow$ (ii) and the $\tau$-function representation $(0.10)$ of $\Psi$ and $\Psi^{*}$, shown in Theorem 0.1 ; this establishes Proposition 3.1.

With each component of the wave vector $\Psi$, given in $(0.10)$, or, what is the same, with each component of the $\tau$-vector, we associate a sequence of infinite-dimensional planes in the Grassmannian $G r^{(n)}$

$$
\begin{aligned}
\mathcal{W}_{n} & =\operatorname{span}_{\mathbf{C}}\left\{\left(\frac{\partial}{\partial t_{1}}\right)^{k} \Psi_{n}(t, z), \quad k=0,1,2, \ldots\right\} \\
& =e^{\sum_{1}^{\infty} t_{i} z^{i}} \operatorname{span}_{\mathbf{C}}\left\{\left(\frac{\partial}{\partial t_{1}}+z\right)^{k} \psi_{n}(t, z), \quad k=0,1,2, \ldots\right\} \\
& =: e^{\sum_{1}^{\infty} t_{i} z^{i}} \mathcal{W}_{n}^{t} .
\end{aligned}
$$

and planes

$$
\mathcal{W}_{n}^{*}=\operatorname{span}_{\mathbf{C}}\left\{\frac{1}{z}\left(\frac{\partial}{\partial t_{1}}\right)^{k} \Psi_{n-1}^{*}(t, z), \quad k=0,1,2, \ldots\right\},
$$

which are the orthogonal complements of $\mathcal{W}_{n}$ in $G r^{(n)}$, by the residue pairing

$$
\langle f, g\rangle_{\infty}:=\oint_{z=\infty} f(z) g(z) \frac{d z}{2 \pi i} .
$$

Proposition 3.2 (ii) $\Longleftrightarrow(i v) \Longleftrightarrow(v)$ holds. 
Adler-van Moerbeke:Discrete KP August 24, 1998 §3, p.21

Proof: The inclusion ... $\supset \mathcal{W}_{n-1} \supset \mathcal{W}_{n} \supset \mathcal{W}_{n+1} \supset \ldots$ in (iv) implies that $\mathcal{W}_{n}$, given by $(3.1)$ and $(0.10)$, is also given by

$$
\mathcal{W}_{n}=\operatorname{span}_{\mathbf{C}}\left\{\Psi_{n}(t, z), \Psi_{n+1}(t, z), \ldots\right\} .
$$

Moreover the inclusions $\ldots \supset \mathcal{W}_{n} \supset \mathcal{W}_{n+1} \supset \ldots$ imply, by orthogonality, the inclusions $\ldots \subset \mathcal{W}_{n}^{*} \subset \mathcal{W}_{n+1}^{*} \subset \ldots$, and thus $\mathcal{W}_{n}^{*}$, given by (3.2) and (0.10) and thus specified by $\Psi_{n-1}^{*}$ and $\tau_{n}$, is also given by

$$
\mathcal{W}_{n}^{*}=\left\{\frac{\Psi_{n-1}^{*}(t, z)}{z}, \frac{\Psi_{n-2}^{*}(t, z)}{z}, \ldots\right\} .
$$

The formula (0.10) for $\Psi_{n}$ and $\Psi_{n-1}^{*}$ imply the bilinear identities (1.1), since each $\tau_{n}$ is a $\tau$-function, yielding $\mathcal{W}_{n}^{*}=\mathcal{W}_{n}^{\perp}$, with respect to the residue pairing and so:

$$
\left\langle\Psi_{n}(t, z), \frac{\Psi_{n-1}^{*}\left(t^{\prime}, z\right)}{z}\right\rangle_{\infty}=\oint_{z=\infty} \Psi_{n}(t, z) \Psi_{n-1}^{*}\left(t^{\prime}, z\right) \frac{d z}{2 \pi i z}=0 .
$$

Since

$$
\mathcal{W}_{n} \subset \mathcal{W}_{m+1}=\left(\mathcal{W}_{m+1}^{*}\right)^{*}, \quad \text { all } n>m
$$

we have the orthogonality $\mathcal{W}_{n} \perp \mathcal{W}_{m+1}^{*}$ for all $n>m$, with respect to the residue pairing; since $\Psi_{n}(t, z) \in \mathcal{W}_{n}, \frac{\Psi_{m}^{*}\left(t^{\prime}, z\right)}{z} \in \mathcal{W}_{m+1}^{*}\left(t^{\prime}, z\right)$, we have

$$
0=\left\langle\Psi_{n}(t, z), \frac{\Psi_{m}^{*}\left(t^{\prime}, z\right)}{z}\right\rangle_{\infty}=\oint_{z=\infty} \Psi_{n}(t, z) \Psi_{m}^{*}\left(t^{\prime}, z\right) \frac{d z}{2 \pi i z}, \quad \text { all } n>m,
$$

which is (ii).

Now assume (ii); then, for fixed $n>m$, we have

$$
0=\oint_{z=\infty}\left(\frac{\partial}{\partial t_{1}}\right)^{k} \Psi_{n}(t, z)\left(\frac{\partial}{\partial t_{1}^{\prime}}\right)^{\ell} \Psi_{m}^{*}\left(t^{\prime}, z\right) \frac{d z}{2 \pi i z}, \quad n>m
$$

and thus by (3.1) and (3.2),

$$
\mathcal{W}_{n} \subseteq\left(\mathcal{W}_{m+1}^{*}\right)^{*}=\mathcal{W}_{m+1}, \quad \text { for } n>m,
$$

which implies the flag condition $\ldots \supset \mathcal{W}_{n-1} \supset \mathcal{W}_{n} \supset \mathcal{W}_{n+1} \supset \ldots$, stated in (iv).

(iv) $\Longleftrightarrow(\mathrm{v})$, follows from the equivalence of (i) and (iii) in Proposition 1.1 , by setting $\tau_{1}:=\tau_{n-1}, \tau_{2}=\tau_{n}, \tilde{\mathcal{W}}_{1}=z^{-n+1} \mathcal{W}_{n-1}$ and $\tilde{\mathcal{W}}_{2}=z^{-n} \mathcal{W}_{n}$ and noting

$$
z\left(z^{-n} \mathcal{W}_{n}\right) \subset\left(z^{-n+1} \mathcal{W}_{n-1}\right), \quad \text { i.e. } \mathcal{W}_{n} \subset \mathcal{W}_{n-1},
$$

concluding the proof of the proposition. 
Adler-van Moerbeke:Discrete KP August 24, $1998 \quad \S 3$, p.22

Proposition $3.3(v) \Longleftrightarrow$ (vi) holds.

Proof:

Step 1. For a given $n \in \mathbf{Z}$, statement (v), namely

$$
R_{k}^{(n)}:=\left\{p_{k-1}(-\tilde{\partial}) \tau_{n}, \tau_{n+1}\right\}+\tau_{n+1} p_{k}(-\tilde{\partial}) \tau_{n}-\tau_{n} p_{k}(-\tilde{\partial}) \tau_{n+1}=0, \quad k \geq 2
$$

implies

$$
R_{k}^{(n)^{\prime}}=\left(\frac{\partial}{\partial t_{k}}-p_{k}(\tilde{\partial})\right) \tau_{n+1} \circ \tau_{n}=0, \quad k \geq 2 .
$$

Since $R_{k}^{(n)}$ are the Taylor coefficients of relation (v) in Theorem 0.2, statement $(\mathrm{v})_{n}$ is equivalent to $(\mathrm{iv})_{n}$ (i.e. $\left.\mathcal{W}_{n} \supset \mathcal{W}_{n+1}\right)$. The latter is equivalent to the bilinear identity (iii) $)_{n}$ (i.e., (0.18) with $n \rightarrow n+1$ and $m \rightarrow n-1$ ). According to the arguments used in the proof of Theorem 0.1, (iii) $)_{n}$ implies $R_{k}^{(n)^{\prime}}=0$.

Step 2. The converse holds, because, upon using an inductive argument,

$$
R_{k}^{(n)}=\alpha R_{k}^{(n)^{\prime}}+\text { partials of }\left(R_{1}^{(n)^{\prime}}, \ldots, R_{k-1}^{(n)^{\prime}}\right) ;
$$

thus the vanishing of the $R_{1}^{(n)^{\prime}}, \ldots, R_{k}^{(n)^{\prime}}$ implies the vanishing of $R_{k}^{(n)}$.

Theorem 3.4 Every 1-Toda lattice is equivalent to a 2-Toda lattice.

Proof: The 1-Toda theory implies for $S_{1}:=S \in I+\mathcal{D}_{-}, L_{1}:=L$

$$
\frac{\partial S_{1}}{\partial t_{n}}=-\left(L_{1}^{n}\right)_{-} S_{1}(t), \quad \text { where } L_{1}=S_{1} \Lambda S_{1}^{-1} .
$$

Then, in view of the 2-Toda theory, define $S_{2}(t) \in \mathcal{D}_{+}$by means of the differential equations

$$
\frac{\partial S_{2}(t)}{\partial t_{n}}=\left(L_{1}^{n}\right)_{+} S_{2}(t), \quad n=1,2, \ldots
$$

with initial condition $S_{2}(0)=\left(\right.$ an invertible element $\left.d_{+} \in \mathcal{D}_{+}\right)$. Then defind $S_{1,2}(t, s)$ and $L_{1,2}=S_{1,2} \Lambda^{ \pm 1} S_{1,2}^{-1}$, flowing according to the commuting differential equations

$$
\frac{\partial S_{1,2}(t, s)}{\partial s_{n}}= \pm\left(L_{2}^{n}(t, s)\right)_{\mp} S_{1,2}(t, s) \quad \text { with } \quad S_{1,2}(t, 0)=S_{1,2}(t) .
$$

\footnotetext{
${ }^{8}$ The first index in $L_{1,2}$ and $S_{1,2}$ corresponds to the upper-sign.
} 
Adler-van Moerbeke:Discrete KP August 24, 1998 §4, p.23

$S_{1,2}(t, s)$ satisfies the $t$-equations of 2-Toda for $s=0$, by construction; now we must check that this holds for $s \neq 0$; therefore, set

$$
F_{1,2}^{(n)}(t, s)=\frac{\partial S_{1,2}}{\partial t_{n}}(t, s) \pm\left(L_{1}^{n}(t, s)\right)_{\mp} S_{1,2}(t, s), \quad \text { for } n=1,2, \ldots
$$

Compute, using (3.5) and $\left[\partial / \partial t_{n}, \partial / \partial s_{n}\right]=0$, the system of two differential equations

$$
\frac{\partial F_{1,2}^{(n)}}{\partial s_{k}}(t, s)= \pm\left[F_{2,1}^{(n)} S_{2}^{-1}, L_{2}^{k}\right]_{\mp} S_{1,2} \pm\left(L_{2}^{k}\right)_{\mp} F_{1,2}^{(n)}, \quad k, n=1,2, \ldots ;
$$

since $F_{1,2}^{(n)}(t, 0)=0$, we have $F_{1,2}^{(n)}(t, s)=0$ for all $s$. Thus, by (3.5) and (3.6), $S_{1,2}(t, s)$ flow according to 2-Toda.

\section{Discrete KP-solutions generated by vertex operators}

An important construction leading to Toda solutions is contained in Theorem 0.3, which is based on the following Lemma:

Lemma 4.1 Particular solutions to equation

$$
\left\{\tau_{1}\left(t-\left[z^{-1}\right]\right), \tau_{2}(t)\right\}+z\left(\tau_{1}\left(t-\left[z^{-1}\right]\right) \tau_{2}(t)-\tau_{2}\left(t-\left[z^{-1}\right]\right) \tau_{1}(t)\right)=0
$$

are given, for arbitrary $\lambda \in \mathbf{C}^{*}$, by pairs $\left(\tau_{1}, \tau_{2}\right)$, defined by:

$$
\tau_{2}(t)=\left(\int X(t, \lambda) \nu(\lambda) d \lambda\right) \tau_{1}(t)=\int e^{\sum t_{i} \lambda^{i}} \tau_{1}\left(t-\left[\lambda^{-1}\right]\right) \nu(\lambda) d \lambda,
$$

or

$$
\tau_{1}(t)=\left(\int X(-t, \lambda) \nu^{\prime}(\lambda) d \lambda\right) \tau_{2}(t)=\int e^{-\sum t_{i} \lambda^{i}} \tau_{2}\left(t+\left[\lambda^{-1}\right]\right) \nu^{\prime}(\lambda) d \lambda .
$$

Proof: Using

$$
e^{-\sum_{1}^{\infty} \frac{1}{i}\left(\frac{\lambda}{z}\right)^{i}}=1-\frac{\lambda}{z}
$$


it suffices to check, before even integrating, that $\tau_{2}(t)=X(t, \lambda) \tau_{1}(t)$ satisfies the above equation (4.1)

$$
\begin{aligned}
e^{-\sum t_{i} \lambda^{i}} & \left(\left\{\tau_{1}\left(t-\left[z^{-1}\right]\right), \tau_{2}(t)\right\}+z\left(\tau_{1}\left(t-\left[z^{-1}\right]\right) \tau_{2}(t)-\tau_{2}\left(t-\left[z^{-1}\right]\right) \tau_{1}(t)\right)\right) \\
= & e^{-\sum t_{i} \lambda^{i}}\left\{\tau_{1}\left(t-\left[z^{-1}\right]\right), e^{\sum t_{i} \lambda^{i}} \tau_{1}\left(t-\left[\lambda^{-1}\right]\right)\right\} \\
& \left.\quad+z\left(\tau_{1}\left(t-\left[z^{-1}\right]\right) \tau_{1}\left(t-\left[\lambda^{-1}\right]\right)-\left(1-\frac{\lambda}{z}\right) \tau_{1}(t) \tau_{1}\left(t-\left[z^{-1}\right]-\lambda^{-1}\right]\right)\right) \\
= & \left\{\tau_{1}\left(t-\left[z^{-1}\right]\right), \tau_{1}\left(t-\left[\lambda^{-1}\right]\right)\right\} \\
\quad & \quad+(z-\lambda)\left(\tau_{1}\left(t-\left[z^{-1}\right]\right) \tau_{1}\left(t-\left[\lambda^{-1}\right]\right)-\tau_{1}(t) \tau_{1}\left(t-\left[z^{-1}\right]-\left[\lambda^{-1}\right]\right)\right) \\
= & 0
\end{aligned}
$$

using the differential Fay identity (1.3) for the $\tau$-function $\tau_{1}$; a similar proof works for the second solution, given by $\tau_{1}(t)=X(-t, \lambda) \tau_{2}(t)$. Since equation (4.1) is linear in $\tau_{1}(t)$, and also in $\tau_{2}(t)$, the equation remains valid after integrating with regard to $\lambda$.

Proof of Theorem 0.3: Note, from the definition of $\tau_{ \pm n}$ in Theorem 3, that each $\tau_{n}$ is defined inductively by

$$
\tau_{n+1}=\int X(t, \lambda) \nu_{n}(\lambda) d \lambda \tau_{n} \text { and } \tau_{-n-1}=\int X(-t, \lambda) \nu_{-n-1}(\lambda) d \lambda \tau_{-n} ;
$$

thus by Lemma 4.1, the functions $\tau_{n+1}$ and $\tau_{n}$ are a solution of equation (v) of Theorem 0.2. Therefore, theorem 0.2 implies that the $\tau_{n}$ 's form a $\tau$-vector of the discrete KP hierarchy.

\section{Example of vertex generated solutions: the $q$-KP equation}

Consider the class of $q$-pseudo-difference operators, with $y$-dependent coefficients, acting on functions $f(y)$

$$
\mathcal{D}_{q}=\left\{\sum a_{i}(y) D^{i}\right\}, \text { with } D f(y):=f(q y) .
$$

and the $q$-derivative $D_{q}$, defined by

$$
D_{q} f(y):=\frac{f(q y)-f(y)}{(q-1) y}=-\lambda(y)(D-1) f(y), \text { with } \lambda(y):=-\frac{1}{(q-1) y}
$$


Adler-van Moerbeke:Discrete KP August 24, $1998 \quad \S 5$, p.25

Consider the following $q$-pseudo-difference operators

$Q=D+u_{0}(x) D^{0}+u_{-1} D^{-1}+\ldots$ and $\quad Q_{q}=D_{q}+v_{0}(x) D_{q}^{0}+v_{-1}(x) D_{q}^{-1}+\ldots$

and the following $q$-deformations, which were proposed respectively by E. Frenkel [6] and Khesin, Lyubashenko and Roger [10], for $n=1,2, \ldots$ :

$$
\begin{array}{lc}
\frac{\partial Q}{\partial t_{n}}=\left[\left(Q^{n}\right)_{+}, Q\right] & \text { (Frenkel system) } \\
\frac{\partial Q_{q}}{\partial t_{n}}=\left[\left(Q_{q}^{n}\right)_{+}, Q_{q}\right], & \text { (KLR system) }
\end{array}
$$

where ()$_{+}$and ()$_{-}$refer to the $q$-difference and strictly $q$-pseudo-differential part of ( ). Define

$c(x)=\left(\frac{(1-q) x}{1-q}, \frac{(1-q)^{2} x^{2}}{2\left(1-q^{2}\right)}, \frac{(1-q)^{3} x^{3}}{3\left(1-q^{3}\right)}, \ldots\right) \in \mathbf{C}^{\infty} \quad$ and $\quad \lambda_{n}^{-1}=(1-q) x q^{n-1}$,

one checks for $n \geq 1, D^{n} \lambda_{0}(x)=\lambda_{n}(x)$, and

$$
\begin{aligned}
D^{n} c(x) & =c(x)-\sum_{1}^{n}\left[\lambda_{i}^{-1}(x)\right] \\
D^{-n} c(x) & =c(x)+\sum_{1}^{n}\left[\lambda_{-i+1}^{-1}(x)\right]
\end{aligned}
$$

Details about these theorems were reported in a joint work with E. Horozov [4].

Theorem 5.1 There is an algebra isomorphism

$$
\wedge: \mathcal{D}_{q} \longrightarrow \mathcal{D},
$$

which maps the Frenkel and KLR system into the discrete KP-hierarchy

$$
\frac{\partial L}{\partial t_{n}}=\left[\left(L^{n}\right)_{+}, L\right], \quad n=1,2, \ldots
$$


Adler-van Moerbeke:Discrete KP August 24, 1998 §5, p.26

Theorem 5.2 The matrices

$$
L=\Lambda+\sum_{-\infty<\ell \leq 0} \operatorname{diag}\left(\frac{p_{1-\ell}(\tilde{\partial}) \tau_{n+\ell+1} \circ \tau_{n}}{\tau_{n+\ell+1} \tau_{n}}\right)_{n \in \mathbf{Z}} \Lambda^{\ell}
$$

and

$$
\tilde{L}=\varepsilon L \varepsilon^{-1}
$$

with $\varepsilon$ as in (5.11), $\tau_{0}=\tau(c(x)+t)$ and

$$
\begin{aligned}
\tau_{n} & =X\left(t, \lambda_{n}\right) \ldots X\left(t, \lambda_{1}\right) \tau(c(x)+t) \\
& =r_{n}(\lambda)\left(\prod_{k=1}^{n} e^{\sum_{i=1}^{\infty} t_{i} \lambda_{k}^{i}}\right) D^{n} \tau(c(x)+t) \\
\tau_{-n}= & X\left(-t, \lambda_{-n+1}\right) \ldots X\left(-t, \lambda_{0}\right) \tau(c(x)+t) \\
= & r_{-n}(\lambda)\left(\prod_{k=1}^{n} e^{-\sum_{i=1}^{\infty} t_{i} \lambda_{-k+1}^{i}}\right) D^{-n} \tau(c(x)+t)
\end{aligned}
$$

transform, using the map , respectively into solutions to the $q$-KP deformations (5.1) and (5.2) of

$$
Q=D+\sum_{-\infty<i \leq 0} a_{i}(y) D^{i} \quad \text { or } \quad Q_{q}=D_{q}+\sum_{-\infty<i \leq 0} b_{i}(y) D_{q}^{i}
$$

where the $b_{i}$ are related to the $a_{i}$ by (5.12) and?

$a_{\ell}(y)=$ polynomial in $\left\{\begin{array}{l}\frac{\partial^{k}}{\partial t_{i_{1}} \ldots \partial t_{i_{k}}} \log \left(\tau(c(y)+t)^{\pi(k)} D^{\ell+1} \tau(c(y)+t)\right) \text { for } k \geq 2 \\ \sum_{i=1}^{\ell+1} \lambda_{i}^{j}(y)+\frac{\partial}{\partial t_{j}} \log \frac{D^{\ell+1} \tau(c(y)+t)}{\tau(c(y)+t)}, \text { for } k=1\end{array}\right.$

The proofs of these theorems, which rely heavily on the next lemma, will be given later. In an elegant recent paper, Iliev 9] has obtained $q$-bilinear identities and $q$-tau functions, as well, purely within the KP theory.

\footnotetext{
${ }^{9} \pi(k)=$ parity of $k=1$, when $k$ is even, and $=-1$, when $k$ is odd.
} 
Adler-van Moerbeke:Discrete KP August 24, $1998 \quad \S 5$, p.27

Consider an appropriate space of functions $f(y)$ representable by "Fourier" series

$$
f(y)=\sum_{-\infty}^{\infty} f_{n} \varphi_{n}(y)
$$

in the basis $\varphi_{n}(y):=\delta\left(q^{-n} x^{-1} y\right)$ for fixed $q \neq 1$, and a parameter $x \in \mathbf{R}$. Also, remember

$$
\lambda_{i}:=D^{i} \lambda_{0}=\lambda\left(x q^{i}\right)
$$

Lemma 5.3 Then the Fourier transform,

$$
f \longmapsto \mathcal{F} f=\left(\ldots, f_{n}, \ldots\right)_{n \in \mathbf{Z}}
$$

induces an algebra isomorphism^, mapping D-operators into $\Lambda$-operators

$$
\begin{aligned}
\mathfrak{D}_{q} & \longrightarrow \mathcal{D} \\
\sum_{i} a_{i}(y) D^{i} & \longmapsto \sum \hat{a}_{i} \Lambda^{i}:=\sum_{i} \operatorname{diag}\left(\ldots, a_{i}\left(x q^{n}\right), \ldots\right)_{n \in \mathbf{Z}} \Lambda^{i} .
\end{aligned}
$$

Moreover

$$
\sum_{i=0}^{n} b_{i}(y) D_{q}^{i}=\sum_{i=0}^{n} a_{i}(y)(-\lambda D)^{i} \longmapsto \varepsilon\left(\sum_{i=0}^{n} \hat{a}_{i} \Lambda^{i}\right) \varepsilon^{-1},
$$

where the $\Lambda$-operator in brackets is monic, with

$$
\begin{gathered}
\hat{\lambda}=\left(\ldots, \lambda_{-1}(x), \lambda_{0}(x), \lambda_{1}(x), \ldots\right)=\left(\ldots, D^{-1} \lambda, \lambda, D \lambda, \ldots\right) \\
\varepsilon:=\operatorname{diag}\left(\ldots, \lambda_{-2} \lambda_{-1},-\lambda_{-1}, 1,-\frac{1}{\lambda_{0}}, \frac{1}{\lambda_{0} \lambda_{1}},-\frac{1}{\lambda_{0} \lambda_{1} \lambda_{2}}, \ldots\right) \text { with } \varepsilon_{0}=1 \\
a_{i}(y):=\sum_{0 \leq k \leq n-i} \frac{\left[\begin{array}{c}
k+i \\
k
\end{array}\right]}{\left(-y(q-1) q^{i}\right)^{k}} b_{k+i}(y) .
\end{gathered}
$$

\footnotetext{
${ }^{10}$ The $\delta$-function $\delta(z):=\sum_{i \in \mathbf{Z}} z^{i}$; enjoys the property $f(z a) \delta(z)=f(a) \delta(z)$

${ }^{11}$ with $[j]:=\frac{1-q^{j}}{1-q}$ and $\left[\begin{array}{l}n \\ k]:=\frac{[n][n-1] \ldots[n-k+1]}{[k][k-1] \ldots[1]}\end{array}\right.$
} 
Adler-van Moerbeke:Discrete KP August 24, $1998 \quad \S 5$, p.28

Proof: The operators $D$ and multiplication by a function $a(y)$ act on basis elements, as follows:

$$
D \varphi_{n}(y)=\varphi_{n-1}(y) \quad \text { and } \quad a(y) \varphi_{n}(y)=a\left(x q^{n}\right) \varphi_{n}(y) .
$$

Therefore $D^{k}$ and $a(y)$ act on functions $f(y)$, as

$$
\begin{aligned}
f(y)=\sum_{n \in \mathbf{Z}} f_{n} \varphi_{n}(y) \longmapsto D^{k} f(y) & =\sum_{n \in \mathbf{Z}} f_{n} D^{k} \varphi_{n}(y) \\
& =\sum_{n \in \mathbf{Z}} f_{n} \varphi_{n-k}(y) \\
& =\sum_{n \in \mathbf{Z}} f_{n+k} \varphi_{n}(y),
\end{aligned}
$$

and

$$
\begin{aligned}
f(y)=\sum_{n \in \mathbf{Z}} f_{n} \varphi_{n}(y) \longmapsto a(y) f(y) & =\sum_{n \in \mathbf{Z}} f_{n} a(y) \varphi_{n}(y) \\
& =\sum_{n \in \mathbf{Z}} f_{n} a\left(x q^{n}\right) \varphi_{n}(y),
\end{aligned}
$$

from which it follows that

$$
\begin{gathered}
\left(D^{k}\right)^{\wedge}=\Lambda^{k} \\
\hat{a}(y)=\operatorname{diag}\left(\ldots, a\left(x q^{n}\right), \ldots\right)_{n \in \mathbf{Z}} .
\end{gathered}
$$

To establish the algebra isomorphism (5.8), one checks that

$$
\begin{aligned}
\left(a(y) D^{i}\right) \wedge\left(b(y) D^{j}\right) \wedge & =\hat{a}(y) \Lambda^{i} \hat{b}(y) \Lambda^{j} \\
& =\hat{a}(y)\left(\Lambda^{i} \hat{b}(y) \Lambda^{-i}\right) \Lambda^{i+j} \\
& =\operatorname{diag}\left(\ldots, a\left(x q^{n}\right) b\left(x q^{n+i}\right), \ldots\right)_{n \in \mathbf{Z}} \Lambda^{i+j} \\
& =\left(a(y) b\left(y q^{i}\right) D^{i+j}\right) \wedge \\
& =\left(a(y) D^{i} b(y) D^{j}\right) \hat{.}
\end{aligned}
$$

Using the inductively established identity

$$
D_{q}^{n}=\frac{1}{y^{n}(q-1)^{n} q^{\frac{n(n-1)}{2}}} \sum_{k=0}^{n}(-1)^{k} q^{k(k-1) / 2}\left[\begin{array}{l}
n \\
k
\end{array}\right] D^{n-k},
$$


Adler-van Moerbeke:Discrete KP August 24, $1998 \quad \S 5$, p.29

the first identity (5.9) is immediate.

Then, using, by virtue of (5.10) and (5.11), $\hat{\lambda} \Lambda=-\varepsilon \Lambda \varepsilon^{-1}$ and $\varepsilon \hat{a} \varepsilon^{-1}=\hat{a}$ (since $\hat{a}$ is diagonal), one computes, using the established isomorphism,

$$
\begin{aligned}
\left(a_{i}(y)(-\lambda(y) D)^{i}\right)^{\wedge} & =\hat{a}_{i}(-\hat{\lambda} \hat{D})^{i} \\
& =\hat{a}_{i}(-\hat{\lambda} \Lambda)^{i} \\
& =\hat{a}_{i}\left(\varepsilon \Lambda \varepsilon^{-1}\right)^{i} \\
& =\varepsilon\left(\hat{a}_{i} \Lambda^{i}\right) \varepsilon^{-1}
\end{aligned}
$$

establishing (5.9).

Proof of Theorem 5.1: Indeed the Frenkel system (5.1) maps at once into $\overline{(5.5), \text { whereas, using }}(5.9)$, the KLR-system maps into

$$
\begin{aligned}
\frac{\partial \varepsilon L \varepsilon^{-1}}{\partial t_{n}} & =\left[\left(\varepsilon L^{n} \varepsilon^{-1}\right)_{+}, \varepsilon L \varepsilon^{-1}\right] \\
& =\varepsilon\left[\left(L^{n}\right)_{+}, L\right] \varepsilon^{-1}
\end{aligned}
$$

which upon conjugation by $\varepsilon$ leads to (5.5) as well.

Proof of Theorem 5.2: From Theorem 0.3, it follows that $L$ with the $\tau_{n}$ 's defined by (5.6), satisfies the Toda lattice; the second equality in (5.6) follows from (5.4). According to Lemma 1.3,

$$
\frac{p_{1-\ell}(\tilde{\partial}) \tau_{n+\ell+1} \circ \tau_{n}}{\tau_{n+\ell+1} \tau_{n}}=\text { a polynomial in }\left(\frac{\partial^{k}}{\partial t_{i_{1}} \ldots \partial t_{i_{k}}} \log \left(\tau_{n+\ell+1} \tau_{n}^{\pi(k)}\right)\right),
$$

where by (5.6), for $k \geq 2$,

$$
\begin{gathered}
\left(\frac{\partial^{k}}{\partial t_{i_{1}} \ldots \partial t_{i_{k}}} \log \left(\tau_{n+\ell+1} \tau_{n}^{\pi(k)}\right)\right)_{n \in \mathbf{Z}} \\
=\left(D^{n} \frac{\partial^{k}}{\partial t_{i_{1}} \ldots \partial t_{i_{k}}} \log \left(\tau(c(y)+t)^{\pi(k)} D^{\ell+1} \tau(c(y)+t)\right)\right)_{n \in \mathbf{Z}} \\
=\left(\frac{\partial^{k}}{\partial t_{i_{1}} \ldots \partial t_{i_{k}}} \log \tau(c(y)+t)^{\pi(k)} D^{\ell+1} \tau(c(y)+t)\right)^{\wedge}
\end{gathered}
$$


Adler-van Moerbeke:Discrete KP August 24, $1998 \quad \S 5$, p.30

and

$$
\begin{aligned}
\left(\frac{\partial}{\partial t_{j}} \log \right. & \left.\frac{\tau_{n+\ell+1}}{\tau_{n}}\right)_{n \in \mathbf{Z}} \\
& =\left(\frac{\partial}{\partial t_{j}} \log \frac{\left(\prod_{\alpha=1}^{n+\ell+1} e^{\sum_{i=1}^{\infty} t_{i} \lambda_{\alpha}^{i}}\right) D^{n+\ell+1} \tau(c(y)+t)}{\left(\prod_{\alpha=1}^{n} e^{\sum_{i=1}^{\infty} t_{i} \lambda_{\alpha}^{i}}\right) D^{n} \tau(c(y)+t)}\right)_{n \in \mathbf{Z}} \\
& =\left(\sum_{\alpha=n+1}^{n+\ell+1} \lambda_{\alpha}^{j}(y)+\frac{\partial}{\partial t_{j}} \log \frac{D^{n+\ell+1} \tau(c(y)+t)}{D^{n} \tau(c(y)+t)}\right)_{n \in \mathbf{Z}} \\
& =\left(D^{n}\left(\sum_{i=1}^{\ell+1} \lambda_{i}^{j}(y)+\frac{\partial}{\partial t_{j}} \log \frac{D^{\ell+1} \tau(c(y)+t)}{\tau(c(y)+t)}\right)\right)_{n \in \mathbf{Z}} \\
& =\left(\sum_{i=1}^{\ell+1} \lambda_{i}^{j}(y)+\frac{\partial}{\partial t_{j}} \log \frac{D^{\ell+1} \tau(c(y)+t)}{\tau(c(y)+t)},\right.
\end{aligned}
$$

establishing Theorem 5.2.

$\underline{\text { Remark: }}$ Note the $\varepsilon$-conjugation has no counterpart in $\mathcal{D}_{q}$-world.

Defining the simple $q$-vertex operators:

$$
X_{q}(x, t, z):=e_{q}^{x z} X(t, z) \quad \text { and } \quad \tilde{X}_{q}(x, t, z):=\left(e_{q}^{x z}\right)^{-1} X(-t, z)
$$

in terms of the vertex operator (6.1) and the $q$-exponential $e_{q}^{x}=e^{\sum_{1}^{\infty} \frac{(1-q)^{k} x^{k}}{k\left(1-q^{k}\right)}}$ we now state:

Corollary 5.4 Any $K$-P $\tau$-function leads to a $q$ - $K$-P $\tau$-function $\tau(c(x)+t)$ satisfying q-bilinear relations below for all $x \in \mathbf{R}, t, t^{\prime} \in \mathbf{C}^{\infty}$ and all $n>m$, which tends to the standard K-P bilinear identity when $q$ goes to 1 :

$$
\begin{gathered}
\oint_{z=\infty} D^{n}\left(X_{q}(x, t, z) \tau(c(x)+t)\right) D^{m+1}\left(\tilde{X}_{q}\left(x, t^{\prime}, z\right) \tau\left(c(x)+t^{\prime}\right) d z=0\right. \\
\longrightarrow \int_{z=\infty} X(t, z) \tau(\bar{x}+t) X\left(t^{\prime}, z\right) \tau\left(\bar{x}+t^{\prime}\right) d z=0 .
\end{gathered}
$$


Adler-van Moerbeke:Discrete KP August 24, $1998 \quad \S 5$, p.31

Proof: The $\tau$-functions $\tau_{n}$ defined in Theorem 5.2 satisfy the usual bilinear identity (0.18), and so, using the following identity

$$
\begin{aligned}
\frac{z^{n-m-1}}{\prod_{k=m+2}^{n}(-\lambda)^{k}} \prod_{k=m+2}^{n} e^{-\sum_{i=1}^{\infty} \frac{1}{i}\left(\frac{\lambda_{k}}{z}\right)^{i}} & =\prod_{k=m+2}^{n}\left(1-\frac{z}{\lambda_{k}}\right) \\
& =\prod_{k=m+2}^{n} e^{-\sum_{i=1}^{\infty} \frac{1}{i}\left(\frac{z}{\lambda_{k}}\right)^{i}} \\
& =D^{n} e_{q}^{x z} D^{m+1}\left(e_{q}^{x z}\right)^{-1}
\end{aligned}
$$

in computing $\tau_{n}\left(t-\left[z^{-1}\right]\right)$ in the usual bilinear identity yields, up to a multiplicative factor $\alpha(\lambda, \nu)$ :

$$
\begin{aligned}
& \alpha(\lambda, \nu) \oint_{z=\infty} \tau_{n}\left(t-\left[z^{-1}\right]\right) \tau_{m+1}\left(t^{\prime}+\left[z^{-1}\right]\right) e^{\sum_{1}^{\infty}\left(t_{i}-t_{i}^{\prime}\right) z^{i}} z^{n-m} \frac{d z}{z} \\
= & \oint_{z=\infty} \tau\left(c(x)+t-\left[z^{-1}\right]-\sum_{1}^{n}\left[\lambda_{i}^{-1}\right]\right) \tau\left(c(x)+t^{\prime}+\left[z^{-1}\right]+\sum_{1}^{m+1}\left[\lambda_{i}^{-1}\right]\right) \\
\prod_{k=m+2}^{n}\left(1-\frac{z}{\lambda_{k}}\right) e^{\sum_{1}^{\infty}\left(t_{i}-t_{i}^{\prime}\right) z^{i}} d z & \oint_{z=\infty} D^{n}\left(X_{q}(x, t, z) \tau(c(x)+t)\right) D^{m+1}\left(\tilde{X}_{q}\left(x, t^{\prime}, z\right) \tau\left(c(x)+t^{\prime}\right)\right) d z=0,
\end{aligned}
$$

the latter tending as $q \rightarrow 1$ to the usual KP bilinear identity, upon using $(5.3)$.

Corollary 5.5 If we take $\tau_{0}(t)=\tau(c(x)+t)$ in Theorem 5.2, with $\tau(t)$ a $N$-KdV $\tau$-function, i.e., $\partial \tau / \partial t_{i N}=0, i=1,2, \ldots$, then

$$
\left(L^{N}\right)=\left(L^{N}\right)_{+} \quad \text { and } \quad \tilde{L}^{N}=\left(\tilde{L}^{N}\right)_{+}
$$

yielding the $N$-Frenkel and $N$-KLR system:

$$
Q^{N}=\left(Q^{N}\right)_{+} \quad \text { and } \quad Q_{q}^{N}=\left(Q_{q}^{N}\right)_{+}
$$

The q-differential operator $Q_{q}^{N}$ has the form below and tends to the differential operator of the $N-K d V$ hierarchy as q goes to 1 :

$Q_{q}^{N}=D_{q}^{N}+\frac{\partial}{\partial t_{1}} \log \frac{\tau\left(D^{N} c+t\right)}{\tau(c+t)} D_{q}^{N-1}$ 
Adler-van Moerbeke:Discrete KP August 24, $1998 \quad \S 5$, p.32

$$
\begin{aligned}
& +\quad \sum_{i=0}^{N-1} \frac{\partial^{2}}{\partial t_{1}^{2}} \log \tau\left(D^{i} c+t\right) \\
& -\quad \sum_{i=0}^{N-2} \lambda_{i+1}\left(\frac{\partial}{\partial t_{1}} \log \frac{\tau\left(D^{N} c+t\right)}{\tau\left(D^{N-1} c+t\right)}-\frac{\partial}{\partial t_{1}} \log \frac{\tau\left(D^{i+1} c+t\right)}{\tau\left(D^{i} c+t\right)}\right) \\
& \left.+\quad \sum_{0 \leq i \leq j \leq N-2} \frac{\partial}{\partial t_{1}} \log \frac{\tau\left(D^{i+1} c+t\right)}{\tau\left(D^{i} c+t\right)} \frac{\partial}{\partial t_{1}} \log \frac{\tau\left(D^{j+1} c+t\right)}{\tau\left(D^{j} c+t\right)}\right) D_{q}^{N-2}+\ldots \\
& \longrightarrow \quad\left(\frac{\partial}{\partial x}\right)^{N}+N \frac{\partial^{2}}{\partial t_{1}^{2}} \log \tau(\bar{x}+t)\left(\frac{\partial}{\partial x}\right)^{N-2}+\ldots
\end{aligned}
$$

Proof: Note that for $W \in G r^{(0)}, z^{N} W \subset W$ if and only if its tau function is of the form $e^{\sum_{1}^{\infty} c_{i} t_{i N}} \tau(t)$, with $\partial \tau(t) / \partial t_{i N}=0, i=1,2, \ldots$. Thus by hypothesis, we have for each

$$
W_{k}=\operatorname{span}\left\{\psi_{k}(t, z), \psi_{k+1}(t, z), \ldots\right\}
$$

$z^{N} W_{k} \subset W_{k}$ and since $L \psi=z \psi$,

$$
z^{N} \psi_{k}=\sum_{j=0}^{N-1} a_{j} \psi_{k+j}+\psi_{k+N}=\left(L^{N} \psi\right)_{k},
$$

and so $L^{N}$ is upper-triangular, yielding (5.21), which by the isomorphism $\wedge$ of Lemma 5.3 yields (5.22). From (0.13) and the relationship between $a_{i}(y)$ and $b_{i}(y)$ given in $(5.12)$, deduce $(5.23)$.

\section{References}

[1] M. Adler and P. van Moerbeke: Birkhoff strata, Bäcklund transformations and limits of isospectral operators , Adv. in Math., 108 140-204 (1994).

[2] M. Adler and P. van Moerbeke: Matrix integrals, Toda symmetries, Virasoro constraints and orthogonal polynomials Duke Math.J., 80 (3), 863-911 (1995)

[3] M. Adler and P. van Moerbeke: Generalized orthogonal polynomials, discrete KP and Riemann-Hilbert problems, Comm. Math. Phys., 207(3), 589-620 (1999) 
Adler-van Moerbeke:Discrete KP August 24, 1998 55, p.33

[4] M. Adler, E. Horozov and P. van Moerbeke: The solution to the $q-K d V$ equation, Phys. Letters A, 242 139-151 (1998).

[5] E. Date, M. Jimbo, M. Kashiwara, T. Miwa: Transformation groups for soliton equations, Proc. RIMS Symp. Nonlinear integrable systems, Classical and quantum theory (Kyoto 1981), pp. 39-119. Singapore : World Scientific 1983.

[6] E. Frenkel: Deformations of the KdV hierarchy and related soliton equations, Int. Math. Res. Notices, 2 55-76 (1996).

[7] D. Gieseker: The Toda hierarchy and the KdV hierarchy , preprint, alg-geom/9509006.

[8] L. Haine and P. Iliev: The bispectral property of a q-deformation of the Schur polynomials and the $q-K d V$ hierarchy, J. of Phys. A: Math. Gen, 30, 7217-7227 (1997).

[9] P. Iliev: Tau-function solutions to a q-deformation of the KP-hierarchy, preprint (1997).

[10] B. Khesin, V. Lyubashenko and C. Roger: Extensions and contractions of the Lie algebra of q-Peudodifferential symbols on the circle, J. of functional analysis, 143 55-97 (1997).

[11] B. A. Kupershmidt: Discrete Lax equations and differential-difference calculus, Astérisque, 123 (1985).

[12] G. Segal, G. Wilson: Loop groups and equations of KdV type, Publ. Math. IHES 61, 5-65 (1985).

[13] K. Takasaki, T. Takebe: Integrable hierarchies and dispersionless limit, Reviews in Math. Phys. 7,743-808 (1995) .

[14] K. Ueno, K. Takasaki: Toda Lattice Hierarchy, Adv. Studies in Pure Math. 4,1-95 (1984) .

[15] P. van Moerbeke: Integrable foundations of string theory, in Lecures on Integrable systems, Proceedings of the CIMPA-school, 1991, Ed.: O. Babelon, P. Cartier, Y. Kosmann-Schwarzbach, World scientific, pp 163-267 (1994). 